Canadian University Music Review

Revue de musique des universités canadiennes

\title{
Le hasard et la technologie chez John Cage : subsistance de la modernité
}

\section{Johanne Rivest}

Volume 21, numéro 2, 2001

URI : https://id.erudit.org/iderudit/1014482ar

DOI : https://doi.org/10.7202/1014482ar

Aller au sommaire du numéro

\section{Éditeur(s)}

Canadian University Music Society / Société de musique des universités canadiennes

ISSN

0710-0353 (imprimé)

2291-2436 (numérique)

Découvrir la revue

Citer cet article

Rivest, J. (2001). Le hasard et la technologie chez John Cage : subsistance de la modernité. Canadian University Music Review / Revue de musique des universités canadiennes, 21(2), 12-28. https://doi.org/10.7202/1014482ar

\section{Résumé de l'article}

Bien que l'on range communément John Cage dans le camp postmoderne, l'auteure entend démontrer que plusieurs traits de la modernité se retrouvent dans sa pratique artistique. Deux aspects principaux en ont été retenus, soit le hasard et la technologie, lesquels se rapportent à la modernité par la manière dont Cage les utilise. D'autres traits de la modernité servent également de points d'ancrage à cette thèse - comme la distanciation objective et l'autonomie de l'œuvre, la critique du convenu en art ou du « musical » en musique, l'idée de progrès ou de changement - à quoi s'opposent certains éléments de l'avant-garde à laquelle Cage se rattache également, paradoxalement.
All Rights Reserved @ Canadian University Music Society / Société de musique des universités canadiennes, 2002
Ce document est protégé par la loi sur le droit d'auteur. L'utilisation des services d'Érudit (y compris la reproduction) est assujettie à sa politique d'utilisation que vous pouvez consulter en ligne.

https://apropos.erudit.org/fr/usagers/politique-dutilisation/ 


\title{
LE HASARD ET LA TECHNOLOGIE CHEZ JOHN CAGE : SUBSISTANCE DE LA MODERNITÉ
}

\author{
Johanne Rivest
}

La pratique artistique de John Cage (1912-1992) est souvent qualifiée de postmoderne, surtout à cause de l'utilisation du hasard et de la grande hétérogénéité des matériaux que celui-ci permet de réunir. Jean-François Lyotard n'hésite d'ailleurs pas à ranger Cage du côté des postmodernes pour l'aspect ludique de son approche ${ }^{1}$. Toutefois, cet essai propose de démontrer que plusieurs des traits de la modernité, déjà adoptés par le Cage d'avant le hasard (1933-1950), subsistent toujours dans sa pratique ultérieure où le hasard domine (à partir de 1951). Parmi ces traits, nous retrouvons la révolte ou le rejet du convenu en art, la distanciation objective et le jeu des formes dénué de recherche expressive pour elle-même ${ }^{2}$, l'immanence du matériau et l'art autonome, sans compter la critique de la modernité incluse dans la modernité même, comme le conçoit Octavio Paz. Pour ce dernier, la postmodernité est englobée par la modernité en tant que critique de celle-ci, aspect qu'il considère inhérent à l'attitude moderne :

Notre critique donne à la tendance nouvelle le nom de " postmodernisme ». Le terme n'est pas très exact. Le soi-disant postmodernisme n'est pas ce qui vient après le modernisme (ce qui vient après est l'avant-garde), c'est une critique du modernisme à l'intérieur du modernisme.

L'art moderne n'est pas seulement le fils de l'âge critique, mais le critique de lui-même ${ }^{3}$.

Octavio Paz ne reconnaît ainsi au terme de postmodernité ${ }^{4}$ aucune légitimation véritable, puisqu'il sous-tendrait un sentiment de révolte caractéristique

1 Jean-François Lyotard, La Condition postmoderne (Paris : Les Éditions de Minuit, 1979), 93, n. 196. Friedemann Sallis abonde dans le même sens dans son article "Le paradoxe postmoderne et l'œuvre tardive de Luigi Nono ", Circuit, musiques contemporaines 11, $\mathrm{n}^{0} 1$ (2000) : 72.

2 À ce sujet, nous pourrions établir une parenté avec le formalisme de Stravinsky, comme l'atteste sa célèbre formule : «[...] je considère la musique par son essence, impuissante à exprimer quoi que ce soit : un sentiment, une attitude, un état psychologique, un phénomène de la nature, etc. L'expression n'a jamais été la propriété immanente de la musique. La raison d'être de celle-ci n'est d'aucune façon conditionnée par celle-là. » Igor Stravinsky, Chroniques de ma vie, nouv. éd. (Paris : Denoël, @1962, 2000), 69-70. C'est l'auteur qui souligne.

3 Octavio Paz, Point de convergence. Du romantisme à l'avant-garde, trad. de l'espagnol par Roger Munier, coll. « Les Essais ", $\mathrm{n}^{\circ} 193$ (Paris : Gallimard, 1976), 132, 16.

$4 \mathrm{Paz}$ utilise le terme postmodernismo, (Octavio Paz, Los hijos del limo [Barcelone : Editorial Seix Barrai, S. A., 1974], 136) traduit justement par postmodernisme dans la version française (Paz, Point 
de la modernité. Plusieurs autres auteurs partagent ce point de vue, dont Louis Dupré, pour qui les principes théoriques de la modernité demeurent à la base des critiques qui lui sont adressées :

\begin{abstract}
De plus, nous faisons encore partie de l'âge moderne; bien que nous puissions nous montrer critiques vis-à-vis de ses principes de base, nous continuons d'en adopter plusieurs. Implicitement, les critiques de la modernité en acceptent davantage certaines hypothèses qu'ils ne parviennent à en écarter. Même ceux qui rejettent la totalité de ses principes théoriques persistent à construire à partir d'eux $x^{5}$.
\end{abstract}

Deux éléments principaux de la pratique cagienne serviront de point d'ancrage à cette démonstration de la subsistance de la modernité. Le premier aspect abordé sera le hasard, dont l'emploi est associé d'emblée à Cage, comme s'il s'agissait de synonymes. Cette association sert souvent de pôle opposé aux méthodes de composition plus rigoureuses issues de la pensée structuraliste. Mais le hasard de Cage connote, paradoxalement, une forme de distanciation objective et de critique de la modernité propres à la modernité même. La technologie est la deuxième notion retenue, car elle est l'un des traits de la modernité qui symbolisent le mieux le rejet du convenu en art et le progrès, comme certitude et comme but. Il y aurait lieu toutefois de nous attarder d'abord à explorer le concept de modernité par quelques-uns des traits qui le sous-tendent.

\title{
DÉFINITIONS
}

Le concept de modernité n'est pas aisé à définir. Le terme lui-même existe depuis plusieurs siècles et son préfixe marque le temps : "modo" signifie en effet « maintenant $»^{6}$. À l'origine, ce terme servait surtout à distinguer ce qui appartenait au présent par opposition à ce qui se rapportait au passé. La notion que le présent surpasse ce qui lui est antérieur semble toutefois plus récente; elle caractérise ce qu'on nomme généralement "l'âge moderne », qui a pris naissance vers le milieu du $\mathrm{XIX}^{\mathrm{e}}$ siècle pour dénoter une manière artistique qui s'insurgeait contre les pratiques conventionnelles courantes.

La révolte est certainement l'un des traits principaux de la modernité et plusieurs mouvements artistiques du début du $\mathrm{XX}^{\mathrm{e}}$ siècle s'y sont abreuvés,

\footnotetext{
de convergence, 132). Pourtant, le terme de postmodernité nous semble plus adéquat pour caractériser ce qui est postmoderne, comme le terme de modernité pour ce qui est moderne. Au sens strict, le terme " modernisme » réfère en effet au phénomène interne au catholicisme du début du $\mathrm{XX}^{\mathrm{e}}$ siècle, où des catholiques s'efforcèrent de combler le hiatus entre les connaissances scientifiques de l'heure et la pratique de la foi. Voir Émile Poulat, «Modernisme », Encycolopcedia Universalis (Paris : Encyclopædia Universalis, 1995), 15:549-52.

5 « Moreover, we are still living in the modern age and, however critical we may be of the principles established at its beginning, we continue to share many of them. Critics of modernity implicitly accept more of its assumptions than they are able to discard. Even those who globally reject its theoretical principles continue to build on them. » Louis Dupré, Passage to Modernity: An Essav in the Hermeneutics of Nature and Culture (New Haven : Yale University Press, 1993), 6. Cette traduction et les suivantes sont de l'auteure du présent article, sauf indication contraire.
}

6Dupré, Passage to Modemity, 145-46. 
principalement les mouvements de l'avant-garde historique, tels le dadaïsme, le surréalisme et le futurisme :

La modernité se révolte contre les fonctions de la tradition qui normalisent [...,] contre tout ce qui est normatif. Cette révolte est une façon de neutraliser les critères de la moralité et de l'utilité. Cette conscience esthétique met perpétuellement en scène un jeu dialectique entre le secret et le scandale public; elle a une fascination morbide pour l'horreur qui accompagne l'acte de profaner, même si elle fuit toujours les résultats triviaux de la profanation?

Un retour en force de la fibre révolutionnaire s'est manifesté durant les années 1950 et 1960, et John Cage, qui connaissait personnellement Marcel Duchamp depuis $1942^{8}$, est considéré comme ayant été le principal promoteur des idées dadaïstes aux États-Unis. Quant aux peintres Jasper Johns et Robert Rauschenberg, des amis proches de Cage, il furent les premiers à être qualifiés de "néo-dadaïstes » au début des années $1950^{9}$.

Un autre trait important de la modernité est l'autonomie de l'art :

En simplifiant beaucoup, je dirais que nous pouvons déceler une tendance, dans l'histoire de l'art moderne, vers une autonomie encore plus grande dans la définition et la pratique de l'art. [...] Finalement, une conception esthétique de l'art est apparue vers le milieu du XIX ${ }^{\mathrm{e}}$ siècle; elle encourageait l'artiste à travailler selon la conscience distincte de l'art pour l'art. L'autonomie de la sphère esthétique pouvait alors devenir un projet délibéré ${ }^{10}$.

L'œuvre autonome ne se justifie ainsi par aucun élément expressif extrinsèque, et la subjectivité du créateur se dissout dans la quête d'un jeu de formes à l'état pur, en réaction contre le symbolisme dans l' art, comme le défend le formaliste Hanslick dans son essai Du beau dans la musique : "Qu'on ne cherche donc pas dans une ouvre musicale l'exposition de quelque état de l'âme ou de quelque histoire de cœur; qu'on se borne à y voir de la musique, et on jouira pleinement, purement, de tout ce qu'elle peut donner ${ }^{11}$. » En peinture, il s' agira

7 « Modernity revolts against the normalizing functions of tradition [...,] against all that is normative. This revolt is one way to neutralize the standards of both morality and utility. This aesthetic consciousness continuously stages a dialectical play between secrecy and public scandal; it is addicted to a fascination with that horror which accompanies the act of profaning, and yet is always in flight from the trivial results of profanation. " Jürgen Habermas, " Modernity-An Incomplete Project ", dans Postmodern Culture, édit. par Hal Foster (Londres et Sydney : Pluto Press, 1985), 5. Voir aussi Paz, Point de convergence, 37.

8 Voir le témoignage de Cage lui-même dans Moira Roth et William Roth, « John Cage on Marcel Duchamp: An Interview », dans Difference/Indifference: Musings on Postmodernism, Marcel Duchamp and John Cage, introd. et textes par Moira Roth (Amsterdam : G+B Arts International, 1998), 71-83.

9 Voir Susan Hapgood, Neo-Dada: Redefining Art, 1958-62, catalogue d'exposition (New York : The American Federation of Arts, Universe Publishing, 1994), 12.

10 « Greatly oversimplifying, I would say that in the history of modern art one can detect a trend towards ever greater autonomy in the definition and practice of art. [...] Finally, around the middle of the nineteenth century an aestheticist conception of art emerged, which encouraged the artist to produce his work according to the distinct consciousness of art for art's sake. The autonomy of the aesthetic sphere could then become a deliberate project [...]. » Habermas, " Modernity-An Incomplete Project », 10.

11 Édouard Hanslick, Du beau dans la musique, trad. par Charles Bannelier, revue et complétée par Georges Pucher, coll. «Musique/Passé/Présent » (Paris : Christian Bourgois éditeur, 1986), 105. 
d'exploiter les formes abstraites, d'étudier leur impact sur la perception, comme s'y appliquent les artistes du Bauhaus dans la première moitié du $\mathrm{XX}^{\mathrm{e}}$ siècle. En musique, le recours à des systèmes mathématiques pourra permettre aux compositeurs qui le souhaitent de fabriquer un objet sonore autonome, qui n'est pas le reflet de leur propre subjectivité et qui n'essaie pas de représenter ou de dépeindre des éléments extérieurs à la musique.

\section{CAGE ET La DistanCIATION OBJECTIVE}

Adhérant à la quête moderniste dès le début de sa carrière, John Cage a grandement subordonné ses compositions à divers systèmes, que ce soit les séries de 25 sons dans les années 1930 (ce qui l'a conduit à étudier auprès d'Arnold Schoenberg, en passant par Adolf Weiss, un disciple de ce dernier), les structures rythmiques de la fin des années 1930 à la fin des années 1940, les diagrammes (charts) au début des années 1950 et les systèmes contingents ensuite, comme les opérations de hasard, les gabarits, les imperfections du papier, les zones temporelles souples (bracket pieces), etc. ${ }^{12}$ Ainsi, les structures rythmiques mises au point par Cage à la fin des années 1930 avaient le pouvoir de tout accueillir, ce qui déniait au contenu son caractère inéluctable, trait que Cage accentuera davantage en incorporant le hasard à sa méthode :

La structure est simple parce qu'elle peut être pensée, raisonnée, mesurée.

C'est une discipline qui, une fois acceptée, à son tour accepte tout [...].

Une structure est comme un pont de nulle part à nulle part et tout le monde peut passer dessus : bruits ou tons, maïs ou blé. Qu'importe ${ }^{13}$ ?

\section{VIE ET ART}

Mais l'adhésion de Cage à cet aspect caractéristique de la modernité n'est pas sans favoriser, paradoxalement, son extrême opposé, soit l'inclusion de la vie dans l'art, comme l'avait prônée avant lui l'avant-garde historique. Cette dernière, en effet, s'est farouchement opposée à l'une des conséquences de l'autosuffisance de l'œuvre d'art, soit celle de son isolement esthétique dans une sphère élevée et séparée de la vie. Les mouvements avant-gardistes sont nés d'une révolte à l'égard de l'œuvre autonome et, pour en briser les remparts, ont décidé d'inclure des éléments puisés à la vie dans l'art. C'est ce qu'exploite Kurt Schwitters, dès le début des années 1920, dans ses Merzbild, ou peintures Merz, qui emploient des matériaux anti-artistiques : « De fait, je n' arrivais pas à comprendre pourquoi de vieux billets, du bois flotté, des cartons de vestiaire, des fils et des sections de roues, des boutons et des vieilleries trouvées dans des greniers ou des monceaux de détritus ne pouvaient servir autant de matériaux appropriés pour peindre que la peinture manufacturée ${ }^{14}$. »

12Voir l'avant-propos de John Cage dans Robert Dunn, John Cage (New York : Henmar Press, 1962), 5.

13John Cage, "Discours sur rien ", dans Silence, discours et écrits par John Cage, trad. par Monique Fong (Paris : Denoël, 1970), 68, 79. Traduction de Cage, « Lecture on Nothing », dans Silence (Middletown, Conn. : Wesleyan University Press, 1961), 111, 124.

14 « I could not, in fact, see the reason why old tickets, driftwood, cloakroom tabs, wires and parts of wheels, buttons and old rubbish found in attics and refuse dumps should not be as suitable a material for painting as the paints made in factories. » Kurt Schwitters, cité dans Hapgood, Neo-Dada: Redefining 
Chez Cage, les deux aspects de vie et d'art coexistent et s'interpénètrent. C'est l'avènement des méthodes de composition intégrant le hasard qui exemplifie cette coexistence des opposés. D'une part, le Cage du début des années 1950 , lorsqu'il délaisse l'idée d'expression et de communication au profit de l'exploitation des sons pour eux-mêmes, c'est-à-dire lorsqu'il permet au hasard de morceler la continuité musicale pour engager l'écoute dans l'instant présent, réaffirme sa croyance, plus ou moins consciemment, en l'autonomie de l'art. Partageant en ceci le point de vue de Boulez pour qui le sérialisme intégral est un outil puissant pour éloigner toute subjectivité dans l'acte compositionnel, Cage élabore des diagrammes complexes pour chacun des paramètres du son dans son œuvre pour piano solo Music of Changes $(1951)^{15}$, à la différence que des opérations de hasard issues du Yi King assurent l'enchaînement d'un moment à l'autre, afin de suspendre encore davantage l'intrusion du moi dans l'objet, de faire en sorte que ce dernier soit pur de toute intervention subjective. En effet, l'utilisation par Cage du Yi King, le livre des transformations ${ }^{16}$, livre d'oracles prisé par des générations de Chinois depuis l'Antiquité et même par de nombreux Occidentaux de nos jours, est symptomatique de la nécessité qu'il éprouve de recourir à un système suffisamment complexe pour que le résultat soit impossible à prévoir et surtout à influencer. De façon générale, Cage utilise le $\mathrm{Yi}$ King en formulant ses propres questions et réponses, et se sert du tirage au sort propre à la méthode de divination du livre d'oracles pour associer fortuitement certaines réponses aux questions. Ces questions et réponses sont élaborées en fonction du matériau disponible, ou conditionnées par lui - ce qui est en soi une façon de nier l'inspiration comme source créatrice pour lui préférer l'immanence du matériau, autre trait du modernisme -, et leur jumelage fortuit provoque de nouvelles relations, potentielles dans le matériau, mais « invisibles », qui resteraient cachées sans le hasard. Ainsi, le matériau demeure premier, car il recèle une infinité de potentialités, mais il est paradoxalement contingent dans son aboutissement, conditionnant ainsi une forme d'indifférence vis-à-vis du contenu ou du sens ${ }^{17}$. Tout peut arriver, il ne s'agit que de fournir des conjonctures nouvelles. Mais cette nouveauté est imprévisible, car elle dépend du hasard qui la fait surgir. C'est comme si le hasard donnait accès à une espèce d'antériorité originelle, avant toute forme de rationalité. Chez Cage, l'utilisation du hasard est à mon avis élevée au rang de système, que l'on pourrait qualifier d'irrationnel, servant à la distanciation du sujet créateur; une sorte de non-but délibéré (purposeful purposelessness ${ }^{18}$ )

Art, 1958-62, 53. La citation provient de « Kurt Schwitters Katalog », dans Merz 20 (Hanovre, mars 1927) : 99-100.

15 Pour la description de l'élaboration de Music of Changes, voir Pierre Boulez et John Cage, Correspondance, documents réunis, présentés et annotés par Jean-Jacques Nattiez (Paris : Christian Bourgois éditeur, 1990), 168-75. Voir également Cage, "Composition as Process: 1. Changes ", dans Silence, 18-34.

16Richard Wilhelm, Yi King, le livre des transformations, préf. et trad. française par Étienne Perrot (Paris : Librairie de Médicis, 1973).

17Moira Roth développe cette idée dans son essai de 1977, «The Aesthetic of Indifference », dans Difference/Indifference: Musings on Postmodernism, Marcel Duchamp and John Cage, 33-47.

18Expression que Cage utilise à de nombreuses reprises, dont dans ce texte de 1957 : «And what 
que Cage rapporte volontiers à ses affinités avec les notions de non-ego et de non-intention qu'il puise au bouddhisme zen. Quant aux systèmes rationnels, ils dépendent d'une subjectivité qui les façonnent, même si leur but est d'éliminer cette subjectivité.

D' autre part, le hasard a toujours été pour Cage une notion qu'il rapprochait de la vie ou de la nature, lesquelles étaient exemptes de déterminations extrinsèques, existaient sans volonté opérante. Le hasard était pour Cage la garantie d'une distanciation féconde, car non basée sur des choix individuels et qui pouvait permettre à l'œuvre de se déployer à la façon d'un organisme vivant, sans intervention humaine. L'attitude à promouvoir en devenait alors une d'acceptation d'un donné extérieur à soi, d'une continuité « trouvée » et non choisie délibérément. L'œuvre, autonome, et se générant en quelque sorte par elle-même grâce aux opérations de hasard, était alors absolument séparée de la subjectivité d'origine, quelque chose dont l'existence se situe totalement (idéalement) en dehors du compositeur. Mais l'objectivité, c'est aussi aborder les sons comme extérieurs à soi; les sons « trouvés » dans l'environnement, les sons extérieurs à sa propre culture ou même à sa capacité auditive, rendus audibles au moyen de l'amplification, comme certains passages de Williams Mix ou de Cartridge Music, dont il sera question plus loin. L'objectivité, en somme, résulte d'un intérêt à l'égard de tout ce qui ne relève pas de goûts subjectifs, de tout ce qui se trouve si les goûts sont écartés du jugement. En recourant au hasard, Cage s'assure que ses préférences n'interviendront pas et, par conséquent, que tout deviendra acceptable parce qu'extérieur à soi, objectif. Il se refuse ainsi à sélectionner, intervenir ou interpréter. L'objectivité devient alors neutre à l'égard du matériau; tout va, à condition de prendre « rien » pour base : « Chaque fois que nous établissons une relation, chaque fois que nous connectons deux termes, nous oublions que nous avons à retourner à zéro, avant de parvenir au terme suivant ${ }^{19}$. $) \mathrm{Ce}$ « rien » implique pour Cage le refus, maintes fois énoncé, de l'improvisation, laquelle reflète immanquablement une subjectivité. Le recours au hasard pour composer est conçu par Cage tel un sommet de neutralité par la contingence extrême de ses résultats. Ainsi, Cage démontre une double affiliation par son utilisation du hasard : celle de l'autonomie de l'œuvre d'art puisée au modernisme, grâce à la distanciation du sujet opérant, et celle de l'avant-gardisme, propre à Dada et ensuite au Néo-Dada du milieu du $\mathrm{XX}^{\mathrm{e}}$ siècle, pour qui le hasard est un moyen d'abolir cette autonomie même, ou du moins de la questionner fondamentalement.

\section{CRITIQUe de LA MOderNité}

L'utilisation du hasard peut donc être perçue comme une critique adressée à l'attitude moderne, laquelle consiste à façonner l'objet idéal par une extrême rigueur des moyens utilisés. La querelle entre Boulez et Cage semble être issue de cette divergence d'opinion, car jamais Boulez n'a voulu admettre comme

is the purpose of writing music? One is, of course, not dealing with purposes but dealing with sounds. [...] A purposeful purposelessness or a purposeless play. This play, however, is an affirmation of life [...]. » Cage, «Experimental Music », dans Silence, 12.

19John Cage, Pour les oiseaux, Entretiens avec Daniel Charles (Paris : Belfond, 1976), 86. 
valide le recours au hasard pour composer, ce qu'il interprétait plutôt comme une abdication de la part du compositeur :

La forme la plus élémentaire de la transmutation du hasard se situerait dans l'adoption d'une philosophie teintée d'orientalisme qui masquerait une faiblesse fondamentale dans la technique de la composition; [...] je qualifierais volontiers cette expérience [...] de hasard par inadvertance ${ }^{20}$.

En défendant le hasard comme instrument obligé de l'élaboration musicale, Cage condamne la rationalité à l'état pur comme unique outil de distanciation. Il préfère y adjoindre les moyens irrationnels, lesquels donnent accès à un univers autrement impossible à appréhender : "Toute tentative d'exclure "l'irrationnel" est irrationnelle. Toute stratégie de la composition qui est entièrement "rationnelle" est irrationnelle à l' extrême ${ }^{21}$. » Et ce geste se trouve en accord avec les pratiques de l'avant-garde historique, qui démontraient un vif intérêt pour les techniques intégrant le fortuit :

Certaines techniques créatrices remplaçaient un logique travail artistique par le hasard (cadavre exquis). Bien des techniques plastiques : décalcomanie, frottage, grattage, s'appuyaient sur l'union du hasard et de l'exploration de la sous-conscience ${ }^{22}$.

Pourtant, chez Cage, le recours au hasard met en échec l'inconscient, car il repose sur des calculs préalables, quoique arbitraires ${ }^{23}$. Cette utilisation proprement cagienne du hasard est à coup sûr paradoxale, car elle fait coexister l'accidentel et le calculé.

Mais dans tout procédé de hasard, le contenu perd en importance. En effet, puisqu'un élément est obtenu de manière contingente, cela suppose que n'importe quel autre élément aurait pu s'y substituer, ce qui dénote une idéologie de l'équivalence des matériaux et, par extension, du caractère futile de la notion de créateur démiurge. Cette sorte de cynisme à l'égard de la création met donc en échec la supposée suprématie du compositeur dans la création d'un produit artistique. Marcel Duchamp, qui a utilisé le hasard à maintes reprises, a aussi remis fondamentalement en cause le rôle de l'artiste créateur en présentant son premier ready-made en 1914, le porte-bouteilles, un objet banal sélectionné dans le but de travestir ses connotations usuelles. Au surplus, il a déclaré qu'avec les ready-mades, il voulait " abaisser le statut de l'artiste dans la société, [...] dé-déifier l'artiste et même [...] éliminer l'art complètement ${ }^{24}$. »

20Pierre Boulez, «Alea », dans Relevés d'apprenti (Paris : Seuil, 1966), 41.

21 Cage, «Précurseurs de la musique moderne », dans Silence, discours et écrits par John Cage, 30, n. 1. Traduction de Monique Fong de Cage, «Forerunners of Modern Music », dans Silence, 62, n. 1.

22Ryszard W. Kluszczynski, «Qu'est-ce que c'est l'avant-garde? », trad. par Krystyna Antkowick, Zagadnienia Rodzajow Literackich 30, $\mathrm{n}^{\circ} 1$ (59) (1987) : 31. C'est l'auteur qui souligne.

23Les archives de John Cage, dont la plus grande partie est déposée à la New York Public Library for the Performing Arts et à la Northwestern University d'Evanston en Illinois, permettent de se rendre compte de l'extrême minutie de ces multiples tirages au sort, à chaque étape décisionnelle.

24 « [...] lower the status of the artist in society, [...] de-deify the artist, and even [...] eliminate art entirely ». Hapgood, Neo-Dada: Redefining Art, 1958-62, 15. 
Cette optique réfère au projet anti-artistique propre à l'avant-garde historique, celle qui se situe au début du $\mathrm{XX}^{\mathrm{e}}$ siècle. Le dadaïsme auquel appartenait Duchamp fut en effet le lieu d'une critique féroce adressée aux institutions artistiques et prôna l'anti-art comme solution aux pratiques esthétiques ambiantes.

\section{NOUVEAUX HORIZONS SONORES}

En accord avec l'attitude moderne qui se révolte contre la normalité établie, John Cage a critiqué l'establishment musical en visant d'abord les sons dits « musicaux », par opposition à ceux qui se rapportent aux «bruits », prolongeant par là le manifeste bruitiste de Luigi Russolo du début du $\mathrm{XX}^{\mathrm{e}}$ siècle $^{25}$. Durant les années 1930, avant son invention du piano préparé et son recours au hasard pour composer, Cage s'est consacré à la percussion. Embauché par la Cornish School de Seattle, de 1938 à 1940, à titre d'accompagnateur pour la danse, d'instructeur et de compositeur, Cage a vite fait de recruter des danseurs et musiciens pour former un groupe de percussionnistes, l'un des premiers du genre à l'époque aux États-Unis. En plus de diriger cet ensemble dans l'exécution de ses propres œuvres pour percussion seule, Cage a commandé à divers compositeurs des œuvres utilisant la même formation, tels Lou Harrison, Henry Cowell, Johanna Maria Beyer et William Russell. À l'instar d'autres pionniers, dont Edgard Varèse, l'aspect propre à la percussion qui intéressait Cage par-dessus tout était la possibilité d'explorer de nouveaux moyens musicaux qui auraient l'avantage de mettre l'accent sur le rythme et le timbre plutôt que sur les hauteurs, une nouveauté dans la musique de concert occidentale de l'époque. Cet intérêt pour le son inouï, que les critiques ont eu beaucoup de mal à admettre, fut assurément partagé par nombre de compositeurs dits « modernes » ou " avant-gardistes ${ }^{26}$. Avant la dissémination de la bande magnétique à la fin des années 1940, l'utilisation élaborée de la percussion s'énonce comme une critique dirigée contre le convenu en art, contre les notions admises de beauté. En affirmant que la libération de la gangue esthétique qui enserre les sons offrira une plus grande palette d'expression, la modernité ne nie pas encore l'expression comme telle, mais les conventions qui la régissent ${ }^{27}$. De la même façon, la première pièce de Cage à employer des procédés électroniques, Imaginary Landscape No. 1 (1939), est perçue par lui comme un élargissement du potentiel acoustique. Toujours au service de la Cornish School de Seattle, Cage avait alors accès à un studio de radio local. Fasciné par ces nouvelles avenues sonores, il a intégré à cette pièce des disques de fréquences stables ou variables, chaque catégorie étant « jouée " par deux interprètes disposant chacun d'un tourne-disque dont la vitesse (variable) était contrôlée par un levier; le reste de l'instrumentation comprenait un piano (aux

\footnotetext{
25Luigi Russolo, L'Art des bruits, textes établis et présentés par Giovanni Lista, trad. par Nina Sparta, coll. Avant-gardes (Lausanne : L'Âge d'Homme, 1975).

26Les termes étaient souvent interchangeables dans les critiques de l'époque.

27 À preuve les titres très évocateurs des œuvres de Cage pendant les années 1940, souvent conçues pour accompagner de la danse, comme ceux-ci : Totem Ancestor, Bacchanale, Tossed As It Is Untroubled, Root of an Unfocus, Mysterious Adventure.
} 
cordes étouffées) et une cymbale. Le tout devait être soit présenté sur les ondes de la radio, sorte de Hörspiel ou pièce radiophonique avant l'heure ${ }^{28}$, soit être gravé sur les sillons d'un disque et diffusé en concert, constituant ainsi un prototype de musique électroacoustique où la reproduction sonore remplace l'exécution en direct. La pièce se situe au moment où Cage essayait avec beaucoup d'effort et de conviction, mais en vain, d'établir un Centre de musique expérimentale ${ }^{29}$. À cette époque, la transition des instruments à percussion vers la musique électroacoustique représentait pour Cage un progrès du matériau et de ses potentialités : "Je crois que la musique pour percussion représente une transition entre la musique qui réfère à un domaine sonore particulier et cette musique électronique du futur qui se rapportera au domaine sonore tout entier ${ }^{30}$.»

En 1952, tout juste après avoir opéré son irréversible virage en faveur de l'utilisation du hasard dans ses procédés de composition, il a institué le Project for Magnetic Tape, en collaboration avec les musiciens Earle Brown et David Tudor, ainsi que les ingénieurs Louis et Bebe Barron, grâce au support financier de Paul et Vera Williams. Cela lui a permis d'élaborer une œuvre sur bande, Williams Mix ${ }^{31}$, courte mais complexe, dont les huit pistes font intervenir un catalogue de cinq à six cents infimes extraits, issus de six catégories de sources sonores : sons de la ville, de la campagne, électroniques, produits manuellement (ou joués sur des instruments, comme la musique de répertoire), produits par le vent (dont des chansons) et sons à peine audibles nécessitant une amplification. Le mode d'assemblage de ces sources, entièrement dérivé des opérations de hasard propres au Yi King, se trouve inscrit sur une partition de 192 pages. Il s'agit en fait davantage d'un canevas ou " diagramme » (diagram), selon l' expression de Cage, qui consiste à indiquer laquelle des catégories utiliser, comment et où couper la bande de chaque catégorie, quels paramètres affecter (hauteur, timbre et intensité) et, pour chacun d'eux, s'ils doivent se poursuivre de façon constante ou variable. Le produit fini se trouve fixé sur bande, ce qui en fait une œuvre close. En effet, le degré de contingence au moment de la diffusion est à peu près nul, contrairement à celui qui prévaut dans l'élaboration de l'œuvre, puisque des opérations de hasard ont servi à sélectionner les extraits et à les présenter dans un certain ordre. Cependant, la partition, ou le canevas, permet d'envisager d'autres élaborations, c'est-à-dire où les sources sonores seraient différentes, bien qu'appartenant aux mêmes

28Bien que l'histoire du Hörspiel en Allemagne soit liée à celle de la radio, ce n'est que vers la fin des années 1960 que le Hörspiel signifiait une production autonome strictement acoustique, sans contenu déterminé, par exemple littéraire ou théâtral, et conçue pour être diffusée par la radio. Voir Klaus Schöning, " On the Development of Acoustic Literature in Radio: the German Hörspiel ", Michigan Quarterly Review 25 (hiver 1986) : 28-39.

29Voir James Pritchett, The Music of John Cage (New York : Cambridge University Press, 1993), 11.

30 «I believe that percussion music represents a transition from music which refers to a particular field of sound to that electronic music of the future which will refer to the entire field of sound. " John Cage, ébauche de lettre, s.d., avant l'été 1940 (Archives de John Cage, Northwestern University, Evanston, Ill.).

31 John Cage, Williams Mix (New York : Henmar Press, 1960). Earle Brown et Christian Wolff ont chacun fait une œuvre électroacoustique dans le même cadre, Octet (Brown) et For Magnetic Tape (Wolff). 
catégories et étant traitées identiquement. L'œuvre oscille donc entre la fermeture et l'ouverture; mais ce qu'il est plus pertinent de remarquer est le quasieffacement de ces sources par la profusion de leur assemblage.

À cet égard, Williams Mix est une œuvre qui brouille ses références et leurs significations intrinsèques, un peu à la manière des collages complexes propres à l'avant-garde, dont les Merzbild de Kurt Schwitters ${ }^{32}$, ou les assemblages du néo-dadaïste Allan Kaprow et les combine paintings ${ }^{33}$ de Robert Rauschenberg. Williams Mix propose un univers objectif, hostile à toute forme de représentation, comme les formes d'art avant-gardistes :

Juxtaposition des significations différentes considérées couramment comme incompatibles; extraction des choses de leurs contextes ordinaires pour les introduire dans des contextes nouveaux; déformation de différents types tels étaient les moyens les plus fréquents de la singularisation. [...] L'attitude négative du nouvel art envers les conventions formelles héritées de l'art traditionnel, de même que l'hostilité pour la représentation (mimesis) contribuaient aussi à l'étrangeté de la forme [...] [laquelle] résultait de l'indépendance et de la domination du procédé dans l'œuvre d'art ${ }^{34}$.

Les sons divers, environnementaux ou au contraire «musicaux », en devenant épurés de toute référence anecdotique, soit à un contexte naturel ou culturel, acquièrent alors une forme de neutralité absolue qui les fait entrer dans une catégorie d'éléments compositionnels premiers. Il devient loisible de créer avec ces éléments une nouvelle forme, empreinte de modernité, c'est-à-dire autonome et qui ne renvoie qu'à elle-même. Toutefois, le tirage au sort des différentes combinaisons et attributs de ces éléments contredit l'absolu de la détermination et l'unicité des résultats que suggère l'œuvre rationnelle de la modernité.

La conception que Cage avait de la technologie à cette époque était partagée par un ensemble de compositeurs, dont Pierre Schaeffer et Herbert Eimert, c'est-à-dire celle d' un instrument neutre, parfaitement adapté à la transmission, à la génération ou à la modification du son, ainsi qu'à l'entreposage de données sonores. Toutefois, cet « instrument " possédait un potentiel de renouvellement du matériau sonore qui séduisait nombre de compositeurs modernes. La technologie ne comportait pas les inconvénients et limitations propres aux intermédiaires humains, ni ceux véhiculés par une pensée musicale perçue comme désuète. Selon Cage, elle devait libérer à la fois les compositeurs et auditeurs « des restrictions de l'échelle dodécaphonique [..., ] des préjugés sur la nature du son "musical" [...,] des limitations rythmiques manuelles [...] et de celles qui sont relatives à l'"équilibre" orchestral et la continuité "musicale" » ${ }^{35}$. Ainsi, le vœu de Cage d'atteindre par cet outil au «domaine sonore

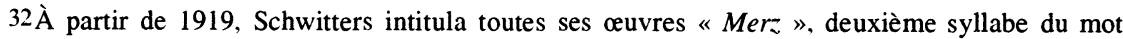
Kommerzbank, apparu fortuitement dans son premier assemblage ou tableau Merz. Les Merzbild ne sont qu'une dénomination générique pour signifier les constructions Merz de Schwitters.

33 Il s'agit de collages visuels, composés d'objets hétéroclites, auxquels se rapportent plusieurs des tableaux de Rauschenberg entre 1953 et 1962.

34Kluszczynski, «Qu'est-ce que c'est l'avant-garde? », 24-25. C'est l'auteur qui souligne. 
tout entier ${ }^{36}$ » se trouvait-il concrétisé. À l'occasion d'un des premiers concerts américains voués exclusivement à la musique électroacoustique internationale, au Festival of Contemporary Arts de l'Université de l'Illinois en 1953, John Cage, l'instigateur du programme, présenta également une communication intitulée « Music for Magnetic Tape: History ». L'extrait suivant démontre bien la foi qu'il portait aux vertus libératrices de la technologie :

Le grand intérêt qu' accordent les compositeurs et même le public à la musique pour bande magnétique dépend des possibilités illimitées qu'elle semble générer. N'importe quel son susceptible d'être enregistré est un matériau potentiel pour cette musique. Tous les sons familiers des instruments musicaux sont disponibles, conjointement avec tous les bruits du quotidien, les sons produits par oscillation électronique et tous les petits sons également, d'amplitude trop basse pour être entendus par l' « oreille nue ». Il est désormais possible de littéralement travailler avec le domaine sonore tout entier, qui n'est plus limité par les hauteurs, les timbres ou les intensités des instruments, ou par les difficultés d'exécution manuelle ou vocale. De même, personne n'est plus contraint par les insuffisances de la notation musicale ${ }^{37}$.

L'une des caractéristiques de la technologie était sa possibilité d'inclure du matériau sans discrimination, car celui-ci pouvait provenir autant de sources traditionnellement "musicales », que de sons puisés à l'environnement qui seraient parfois demeurés inaudibles sans amplification électronique. Ce caractère " généreux » de la technologie la plaçait en quelque sorte au-dessus de l'esprit partisan, en faisait un outil pacifique du progrès, notion éminemment moderne. Le progrès, vu par Cage, était la possibilité de tout inclure, d'être le moins discriminant possible. Cette notion du progrès impliquait une ouverture au tout, dont le " silence » était une métaphore pour Cage. En effet, nous pourrions avancer que le silence procède pour lui d'une sorte d'antériorité originelle, à laquelle le hasard permet une connexion directe et vraie. C'est le non-intentionnel propre à la nature, et le hasard «pige » dans ce domaine sonore tout entier afin de renouveler le matériau. Le hasard s'impose comme un outil pour obtenir des juxtapositions ou superpositions, autrement impossibles à imaginer ou réaliser. Pour Cage, ne l'oublions pas, les fruits du hasard sont supérieurs à ceux de l'improvisation, car ils ne reflètent jamais une

35 « [...] from the limitations of the twelve tone [..., from preconceptions about what constitutes a "musical" sound [...], from manual rhythmic limitations [...], and from limitations about orchestral "balance" and "musical" continuity ». John Cage, description du projet en musique électronique, 1952 (Archives de John Cage, Northwestern University, Evanston, Ill.).

36 Voir note 30.

37 « The great interest that is being accorded music for magnetic tape by composers and audiences alike is due to the fact that the possibilities appear to be virtually unlimited. Any sound that can be recorded is potential material for this music. All the familiar sounds of musical instruments are available together with all the noises of everyday experience, sounds produced by electronic oscillation, and also those small sounds, too low in amplitude to be heard by the "naked ear". One is now literally able to work with the entire field of sound, no longer limited by the pitches of instruments or their timbres or loudnesses, nor by the difficulties of manual or vocal performance. Nor is one any longer bound by the inadequacies of musical notation. » John Cage, « Music for Magnetic Tape: History », 1953 (Archives de John Cage, Northwestern University, Evanston, Ill.). 
subjectivité, étant le résultat d'un procédé qui fonctionne en dehors de toute prédiction.

Continuant dans la même visée, mais désormais imprégnées par l'indétermination, les œuvres de Cage utilisant l'électronique, à partir de la fin des années 1950, remettent l'interprète à l'honneur. L'utilisation du hasard a plusieurs définitions chez Cage. La première consiste à se servir de moyens contingents, en général selon la méthode du tirage au sort propre au Yi King, pour miner le rapport causal entre les questions posées et les réponses obtenues, soit entre les éléments du matériau et leur agencement ${ }^{38}$. Quant à l'indétermination, elle est également une sorte de hiatus entre une proposition et un résultat; toutefois, elle ne résulte pas ici d'un ensemble de questions ou de réponses formulées par le compositeur, mais loge au cœur du rapport compositeur-interprète. Ainsi, une œuvre indéterminée s'énonce comme un potentiel de réalisations multiples, car les spécifications qu'elle coordonne sont minimes et ne touchent pas nécessairement tous les paramètres. L'interprète devra alors élaborer sa propre version, selon les matériaux qu'il aura souvent choisi lui-même et grâce aux spécifications graphiques ou verbales de la partition. Vers le milieu des années 1950, c'est la notion d'inconnu du résultat qui fascine Cage. Dans son texte « Experimental Music: Doctrine », de 1955, il définit ainsi ce qu'il nomme alors "musique expérimentale » et qui explique également sa conception de l'indétermination : « [U]ne action dont le résultat est inconnu $^{39}$. » La non-intention devient ici une autre manière d'innover, car les matériaux se trouvent combinés d'une façon impossible à préétablir rationnellement. Il utilise dès lors les potentialités de l'électronique en direct (live electronics), où l'interprète fait partie intégrante du processus décisionnel. $\mathrm{Au}$ lieu de s'intéresser à la fabrication et à l'entreposage des données sonores, Cage promeut désormais la transformation instantanée du son pendant l'exécution, une manière d'intégrer la vie et ses aléas dans la production artistique, vœu cher aux avant-gardes historiques.

La partition de l'œuvre indéterminée Cartridge Music (1960) demande aux musiciens d'insérer une grande variété d'objets (par exemple, du plastique, des cure-dents, cure-pipes, slinkies, cordes de piano, plumes ou allumettes) dans les têtes de lecture amplifiées de tourne-disques (cartridges); et, pour produire des sons, de frotter, frapper ou gratter ces objets sur différentes surfaces, dont certaines dotées de microphones de contact. Les différentes morphologies sonores obtenues sont immédiatement amplifiées (caractéristique de l'électronique en direct) et le volume de sortie est contrôlé par les interprètes pendant l'exécution, selon une partition graphique dotée d'une grande quantité d'instructions verbales. Chaque exécutant (de un à vingt) doit ainsi constituer sa propre séquence d'activités, selon la lecture faite à partir de cette partition.

Dans l'électronique en direct, l'interprète agit en interaction avec la technologie. Mais celle-ci, de médium neutre qu'elle était (ou à quoi elle prétendait)

38 Voir Johanne Rivest, «Le Concert for Piano and Orchestra de John Cage ou les limites de l'indétermination » (thèse de doctorat, Université de Montréal, 1996), 1:305-17.

39 « $\mathrm{A}] \mathrm{n}$ act the outcome of which is unknown. » Cage, Silence, 13. 
dans la musique électroacoustique du début des années 1950, devient partie prenante de la transmission, colore toute intervention de ses potentialités. Elle agit sur l'information en temps réel et permet au compositeur de montrer les rouages de la mécanique compositionnelle : « Nous envisageons un système sonore tellement flexible qu'un son produit directement sera entendu soit en reproduction fidèle, soit avec ses particularités transformées simultanément en caractéristiques propres à n'importe quel autre son ou encore présenté comme un processus de telles transformation $\mathrm{s}^{40}$. »

Par conséquent, cette nouvelle conception de la technologie propose de présenter un état transitoire, à la manière des éléments biophysiques où la transformation de n'importe quel aspect est incessante, comme dans la nature, ce qui rapproche l'électronique en direct du discours avant-gardiste qui s'érige contre le principe moderne d'autonomie artistique pour favoriser l'inclusion de la vie dans l'art. Ainsi, plutôt que de proposer une œuvre fermée sur elle-même, complète et autonome, cette démonstration du processus de fabrication d'une œuvre se rattache à la manière avant-gardiste d'un Duchamp, par exemple, qui, avec son $\mathrm{Nu}$ descendant un escalier (1912), sectionnait le mouvement en multiples instants superposés. Lors de la création de 0 '00" (4'33" No. 2) en 1962 à Tokyo, Cage s'est également employé à exposer l'élaboration de la pièce, car cette première mondiale a consisté à rédiger les instructions verbales constituant la partition, écriture qui était amplifiée à l'aide de microphones de contact et retransmise en direct à l'auditoire. Le son émis par l'écriture était la pièce, comme si, en voulant brouiller la notion d'œuvre d'art autonome, Cage niait à la fois l'expression et la réification de l'œuvre d'art. Dans le cas de 0'00", l'œuvre n'est que son inscription graphique (du moins lors de la création), qui sert de source sonore amplifiée ${ }^{41}$.

\section{L'ART DÉMYTHIFIÉ}

Cage a fréquemment utilisé l'amplification en direct dans des situations de performance, où le son résultant provenait d'un amalgame de manipulations humaines et de procédés technologiques ${ }^{42}$. C'est l'interaction entre ces deux aspects qui lui a permis d'exacerber la démythification de l'art. Si la constitution interne d'une ouvre d'art dépend d'un ensemble de facteurs contingents tels que réalisés par les interprètes en interaction avec un outillage technologique, la notion d'auteur unique, de produit artistique fermé permettant la contemplation esthétique est alors fortement ébranlée, sinon abolie et remplacée par un processus ouvert d'élaboration, lequel devient à son tour soumis, toutefois, à l'appréciation esthétique. Ainsi, l'avant-gardisme propose de gref-

40 « We envisage a sound system so flexible that a directly produced sound will be heard either faithfully reproduced or with its characteristics simultaneously transformed into those of any other sound or presented as in a process of such transformation or transformations. » Cage, 1963 (Archives de John Cage, Northwestern University, Evanston, Ill.). C'est l'auteur qui souligne.

41 La première phrase des instructions verbales de la partition éditée s'énonce ainsi : "In a situation provided with maximum amplification (no feedback), perform a disciplined action. » John Cage, 0'00" (4'33" No. 2) (New York : Henmar Press, 1962), 1.

42Comme Variations V (1965), Variations VII (1967) ou Reunion (1968). 
fer la vie à l'art en lui soustrayant son caractère sacré et exclusif. Cet objectif passe par la critique de l'institution qui protège l'art, mais pas par son abolition, car l'avant-gardiste en a encore besoin pour promouvoir son art et ses idées :

Les artistes de l'avant-garde n'ont pas cherché à s'isoler, mais plutôt à renouer avec la vie en y intégrant leur art. [...] L'attaque de l'avant-garde contre l'« art » institutionnel de la société bourgeoise visait non seulement à détruire l'institution, mais rendait d'abord visibilité et perceptibilité à son existence et à son importance ${ }^{43}$.

De plus, les éléments constitutifs de ce processus connotent souvent les facteurs triviaux de la vie courante (comme les objets à insérer dans les têtes de lecture de Cartridge Music), plutôt que le raffinement généralement caractéristique des œuvres d'art. En les utilisant, le créateur libère le produit de sa fonction purement artistique et accomplit un lien avec la vie. Cage est l'un des plus ardents promoteurs de l'inclusion des sons banals et quotidiens de l'environnement dans l'art. Paradoxalement, cela les rend susceptibles d'être mis sur un piédestal, d'être assimilés aux objets artistiques offerts à la contemplation, mais sans pourtant les soustraire à leur fonction usuelle dans la vie courante. Cet aspect se rapproche de l'entreprise duchampienne où l' « original »d'un ready-made n'existe pas :

Les ready-mades étaient également une tentative d'éliminer l'« aura » du chefd'œuvre et, en général, de contrecarrer la commodification de l'art. Par nature, les ready-mades ne sont pas uniques; ce sont des objets manufacturés. Comme Duchamp le dira : «Je n'ai jamais eu l'intention de les vendre $[\ldots] »^{44}$.

Même après avoir signé d'innombrables répliques, reproductions ou éditions de ses œuvres en 1963, Duchamp lança à un ami : « Tu sais, j' aime bien signer toutes ces choses - cela les dévalue ${ }^{45}$. " Et Susan Hapgood de commenter : "Il aurait semblé que sa signature eût ajouté de la valeur aux vraies reproductions mais cela aurait certainement dévalué les prétendus "originaux" dont elles étaient les copies ${ }^{46}$.»

43 « Avant-garde artists['] [...] effort was not to isolate themselves, but to reintegrate themselves and their art into life. [...] The avant-garde's attack on the institution "art" in bourgeois society not only was designed to destroy this institution, but permitted its existence and significance to become visible and perceivable in the first place. " Jochen Schulte-Sasse, "Foreword ", dans Peter Bürger, Theory of the Avant-Garde, coll. «Theory and History of Literature », $n^{\circ} 4$ (Minneapolis : University of Minnesota Press, 1984), xxxvi.

44 « The readymades were also an attempt to eliminate the "aura" of the masterpiece, and, in general, to foil the commodification of art. By nature, readymades are not unique; they are manufactured objects. As Duchamp said later, "I never intended to sell them [...]" ". Hapgood, Neo-Dada: Redefining Art, 1958-62, 60, n. 28. Cette citation de Duchamp est tiree de Calvin Tomkins, The Bride and the Bachelors (New York : Penguin Books, 1976), 40.

45 « You know. I like signing all those things-it devalues them. » Cité par Hapgood, Neo-Dada: Redefining Art, 1958-62, 60, n. 30. La citation de Duchamp provient de Tomkins, The Bride and the Bachelors, 68. C'est Tomkins qui souligne.

46 «It would seem that his signing may have added value to the actual reproductions, but of course it would devalue the supposed "originals" of which they were copies. » Hapgood, Neo-Dada: Redefining 


\section{AUTO-CHANGEMENT}

Par l'emploi de la technologie, Cage affirme que l'art peut être inclusif, surtout à l'égard des matériaux conventionnellement rejetés. En fait, l'art devient pour Cage une question d'attitude personnelle, qui ne doit pas être moulée d'après des critères conventionnels. Changer sa manière de percevoir, "se changer » devient dès lors un leitmotiv dans ses actions et propos : « [...] je pense que nous nous trouvons dans une situation plus urgente, où il nous est absolument essentiel de changer fondamentalement d'optique ${ }^{47}$. " En cela, il se démarque des avant-gardistes qui, eux, réclamaient plutôt que ce soit la réalité qui doive changer ${ }^{48}$.

Inspiré par les philosophies orientales, Cage mise ainsi sur l'auto-changement, tant de la part du créateur (lui-même) que de celle des autres intervenants impliqués dans la transmission musicale, y compris les auditeurs. Mais la nécessité de l'auto-changement s'impose parce que les conditions environnantes sont en perpétuel flux et que Cage veut faire « un » avec la vie. Il s'agit donc d'utiliser l'outillage technologique pour se rapprocher de la vie et de se conditionner à en accepter différentes manifestations, quelles qu'elles soient :

[...] nous sommes, en réalité, techniquement outillés pour transformer notre conscience actuelle du mode opératoire de la nature en art $^{49}$.

Nous ne devons pas craindre le futur de la musique.

Mais cette intrépidité advient seulement si, à la croisée des chemins, lorsqu'on constate que les sons se produisent, qu'ils soient voulus ou non, nous nous tournons vers ceux qui ne le sont pas. [...] Ce tournant psychologique conduit au monde de la nature où, graduellement ou subitement, nous percevons que l'humanité et la nature ne sont pas séparés mais coexistent dans ce monde; que rien n'a été perdu lorsque tout fut donné. En fait, tout est gagné ${ }^{50}$.

Cette attitude d'acceptation est en fait une sorte de louange adressée à la nouveauté que fait surgir l'assemblage inédit et fortuit du matériau ou qu'exploite la technologie. En cela, la modernité subsiste toujours chez Cage, par sa croyance aux vertus du changement, corollaire du progrès, et en l'adaptabilité aux conditions environnantes. Son adhésion à la modernité prend toutefois les

Art, $1958-62,60$, n. 30.

47 « [... I think we are in a more urgent situation, where it is absolutely essential for us to change our minds fundamentally. " Cage dans Richard Kostelanetz, Conversing with Cage (New York : Limelight Editions, 1988), 221.

48 « L'avant-garde a surgit de la révolte contre la réalité. [...] Le point de départ des artistes d'avant-garde était simple : si la réalité est mauvaise, si elle ne donne pas à l'homme la possibilité de se réaliser et de vivre la vie digne d'un être humain, il faut la changer. » Kluszczynski, "Qu'est-ce que c'est l'avant-garde? », 28.

49 « [...] we are, in fact, technically equipped to transform our contemporary awareness of nature's manner of operation into art. » Cage, « Experimental Music », 1957, dans Silence, 9.

50 « One need not fear about the future of music. But this fearlessness only follows if, at the parting of the ways, where it is realized that sounds occur whether intended or not, one turns in the direction of those he does not intend. [...] This psychological turning leads to the world of nature, where, gradually or suddenly, one sees that humanity and nature, not separate, are in this world together; that nothing was lost when everything was given away. In fact, everything is gained. » Cage, "Experimental Music », 1957, dans Silence, 8. 
allures d'une critique de la modernité, car Cage prône l'inclusion de la vie et de ses processus constituants dans l'art ou, encore mieux, de l'« art en tant que vie $»^{51}$.

En définitive, l'art n'est plus séparé de la vie, mais c'est la vie qui devient art. Tout est alors susceptible d'être soumis à l'appréciation esthétique, que ce soit les sons produits par des éléments triviaux de la vie courante ou les sons perçus dans l'environnement sans discrimination. La pièce 4'33" (1952) est peut-être celle de Cage qui se rapproche le plus des ready-mades de Duchamp, les trois mouvements minutés servant de socle aux bruits fortuits perçus depuis un lieu circonscrit ${ }^{52}$.

Dans sa recherche de détachement envers le produit artistique, Cage utilise des procédés de hasard, laissant en quelque sorte l'œuvre se constituer objectivement, par le recours à un "système » qui remonte aux origines de la civilisation, le $Y i$ King. L'agencement inédit qu'il obtient ainsi lui assure une réelle nouveauté, celle d'une constitution impossible à appréhender autrement et qui a valeur de vérité, car elle puise à des éléments objectifs par leur isolement de tout contexte symbolique ou signifiant. Ainsi, il est possible d'observer chez John Cage une pérennité de certains des traits de la modernité, bien qu'ils subissent divers travestissements dus à la critique dont ils font l'objet.

\section{Résumé}

Bien que l'on range communément John Cage dans le camp postmoderne, l'auteure entend démontrer que plusieurs traits de la modernité se retrouvent dans sa pratique artistique. Deux aspects principaux en ont été retenus, soit le hasard et la technologie, lesquels se rapportent à la modernité par la manière dont Cage les utilise. D'autres traits de la modernité servent également de points d'ancrage à cette thèse - comme la distanciation objective et l'autonomie de l'œuvre, la critique du convenu en art ou du «musical » en musique, l'idée de progrès ou de changement - à quoi s'opposent certains éléments de l'avantgarde à laquelle Cage se rattache également, paradoxalement.

\section{Abstract}

Although John Cage is usually classed as a post-modernist, this article aims to show that his artistic practice includes several features of modernism. Two main aspects of the latter movement are highlighted: "chance operations" and technology, both related to modernism through the way in which Cage uses them. Other features of modernism underpin this thesis, features such as objective detachment and the autonomy granted to the work, criticism of the traditional in art and of the "musical" in music, the idea of progression or changes-all of which

51 Cage, Pour les oiseaux, 81.

52 Un « socle » aux dimensions variables, puisque les durées des mouvements peuvent varier, tel que le propose la partition éditée par Henmar Press (New York) en $1960:$ « [..] the work may be performed by any instrumentalist or combination of instrumentalists and last any length of time. » 
characteristics of modernism run counter to certain features of avant-gardism, with which, paradoxically, Cage is also linked directly. 